Case Report

Karuna T Karki, MBBS, MS

College of Medical Sciences

Bharatpur, Nepal

Pramod K Chaudhary, MBBS, MS

College of Medical Sciences

Bharatpur, Nepal

Binod Bhattarai, MS, MCh

College of Medical Sciences

Bharatpur, Nepal

Sunil Munakomi, MBBS, MS

College of Medical Sciences

Bharatpur, Nepal

Address for correspondence:

Karuna T Karki, MBBS, MS

College of Medical Sciences

Bharatpur, Nepal

Email: tamrakarkaruna@gmail.com

Received, 13 July, 2015

Accepted, 27 July, 2015

$\mathrm{H}$

arvey Cushing and Walter Dandy are credited with the modern conceptualization of cerebrovascular abnormalities. $^{13}$ Vascular malformations are broadly classified into arteriovenous malformations (AVMs), venous angiomas, cavernous malformations and capillary telangiectasia and arteriovenous fistula (AVF). AVFs are regarded as acquired lesions involving single or multiple arterioles directly connecting with vein without intervening nidus. They are considered as high flow high pressure lesions. Carotid cavernous fistula (CCF), vein of Galen malformation (VOGM) and dural arteriovenous fistula (DAVF) are the example. ${ }^{2,5,14}$ Treatment options for vascular malformations have been evolving with novel techniques in microsurgical, radio surgical and in endovascular therapy. ${ }^{5}$ Before treatment, proper therapeutic decision making is essential. It is a complex process requiring analysis of multiple conduits
Nepal Journal of Neuroscience 12:73-80, 2015

\section{Complex Cerebrovascular Presentation in Central Nepal with Therapeutic Challenge}

Effective management of complex vascular malformation remains one of the more challenging problems faced in most of the developing countries where modern therapeutic resources are deficient. Giant arteriovenous malformations and other vascular malformation like dural arteriovenous malformations are often associated with higher morbidity and mortality rates. These lesions are considered as untreatable or inoperable. Endovascular therapy is an expanding science for managing complex vascular malformations in Nepal that has many constraints. This outset will have positive impact in the country that has to deal with moderate resources to provide wide therapeutic benefit. Here we present our preliminary experience about the use of ethylene vinyl alcohol (Onyx18) to embolize dural arteriovenous fistula and use of n-butyl cyanoacrylate to embolize giant supratentorial arteriovenous malformation.

Key Words: arteriovenous malformation, dural arteriovenous malformation, embolization, ethylene vinyl alcohol, n-butyl cyanoacrylate

of management from the time of diagnosis. Natural history of each vascular malformation should be acknowledged as it can be complicated by number of factors. Clinical presentation, risk of hemorrhage, angioarchitecture and prognosis are often dependent on the types of vascular malformation. ${ }^{13,6}$ Depending upon the complexity of the lesion and clinical presentation, such vascular malformation can be treated with endovascular treatment alone or in combination with microsurgery or radiosurgery as a multimodality treatment. ${ }^{15}$

\section{Case Report}

\section{Case 1}

A 44-year-old right handed healthy gentleman presented with complains of insidious onset of progressive weakness of both lower limbs for a period of one week and that made him difficulty in walking. Couple of days later, 


\section{Karki et al}

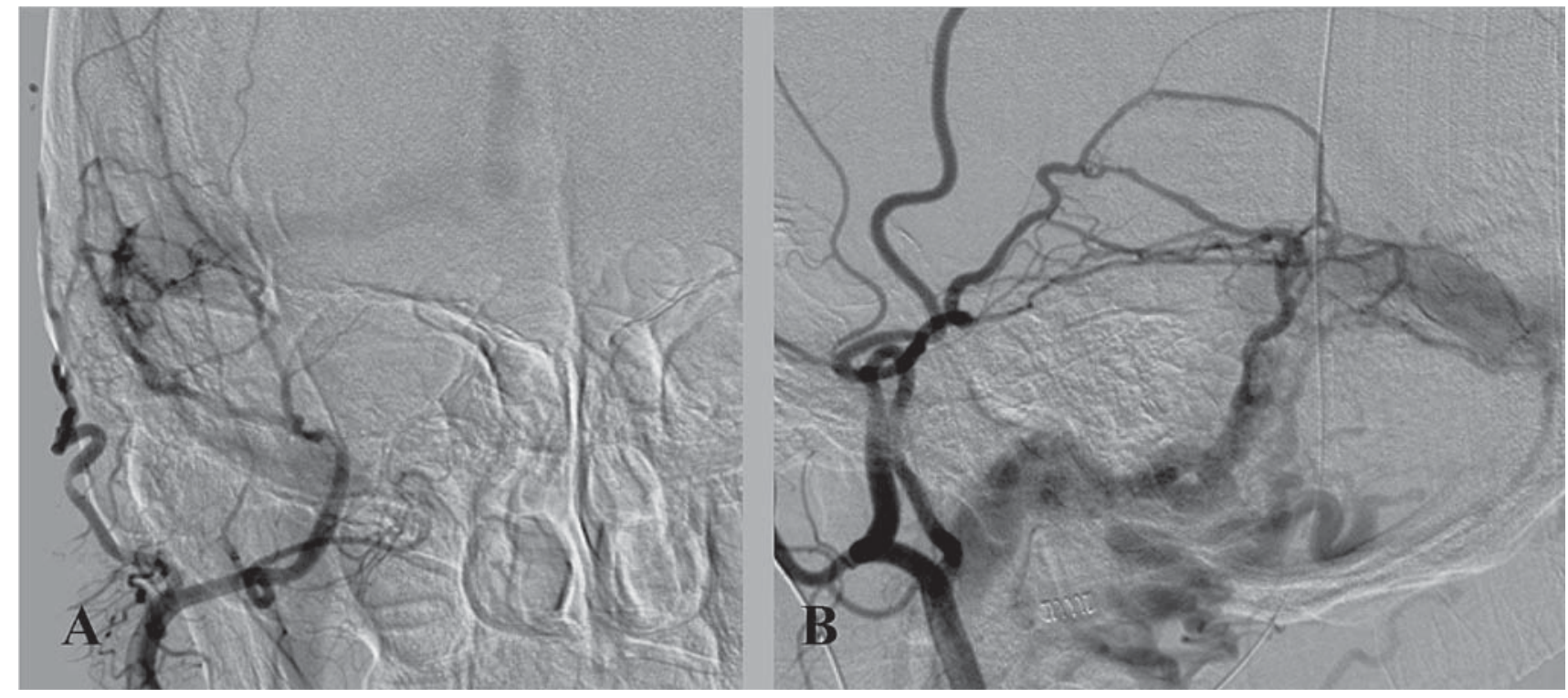

Figure 1: Selective right ECA arteriography showing DAVF supplied by multiple feeders from posterior branch of MMA and draining into right transverse sinus, A) AP view, B) lateral view

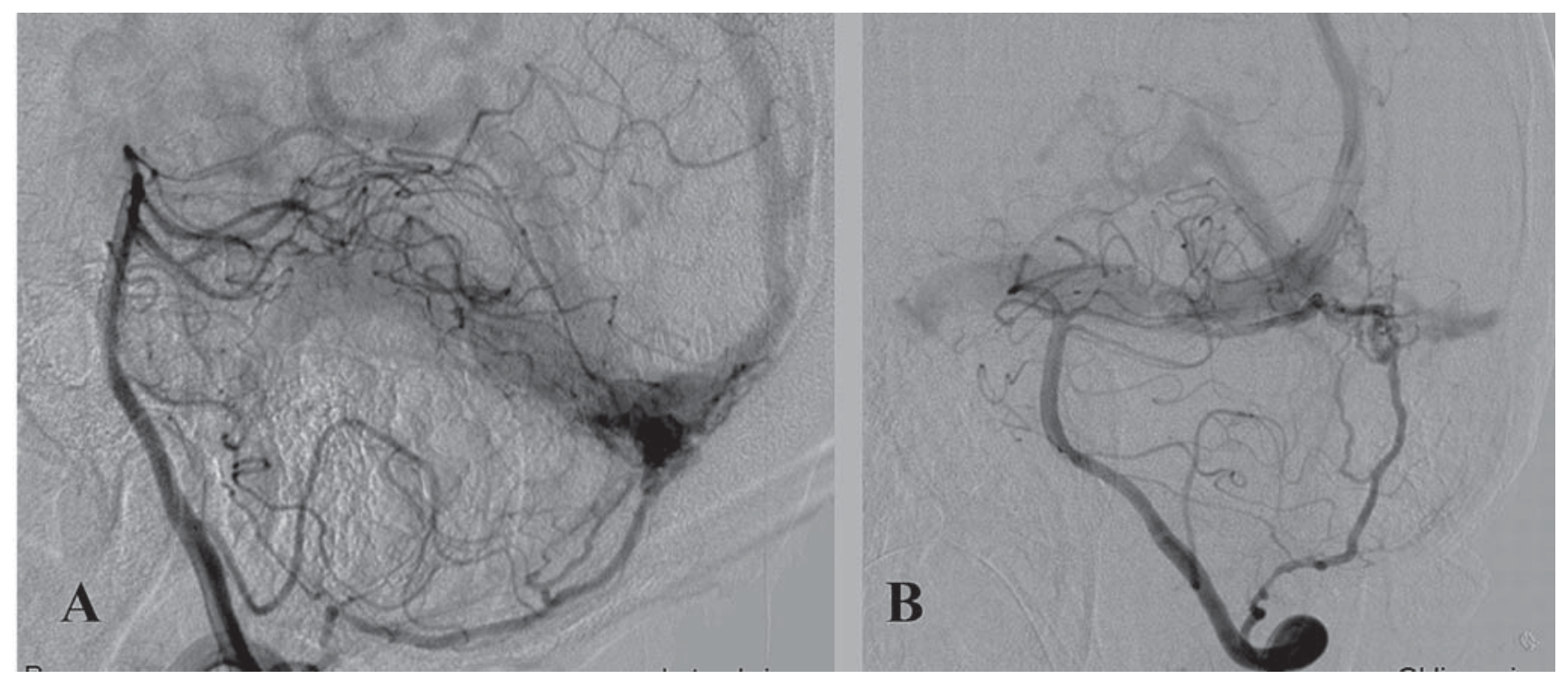

Figure 2: Selective left VA arteriography, DAVF is feeding by posterior meningeal branch of VA and draining into TS, cortical venous reflux is noted, A) lateral view, B) oblique view

he had altered level of consciousness followed by multiple episodes of nausea and vomiting. On examination, his GCS was 11/15. He had paucity in movement in both lower limbs. Power graded as $3+$ in the right and 4 in the left sided extremities. CT head showed abnormally enlarged and tortuous vessels in the subarachnoid space with dilated cortical veins. Patient was admitted in ICU for further angiographic evaluation. 6-vessel angiography revealed Borden grade II non cavernous type DAVF (ncsDAVF). It was supplied by middle meningeal artery (MMA) and occipital branch of right external carotid artery (ECA) (Figure 1A, B), posterior meningeal branch of left vertebral artery (VA) (Figure 2 A, B), and recurrent meningeal branch of right internal carotid artery (ICA) (Figure 3 A). Multiple small tortuous and dural feeders from the left ECA was also noted (Figure 3 B). DAVF was draining majorly into transverse sinus. Retrograde filling of cortical veins (cortical venous reflux) and anterograde filling into dural venous sinuses was observed in early venous phage (Figure 2 A, B).

\section{Technical note}

DSA was performed under uniplanar unit (Toshiba Medical System, Japan) after obtaining Inform consent from both the patients. Under intravenous sedation and 


\section{Cerebrovascular Lesion}

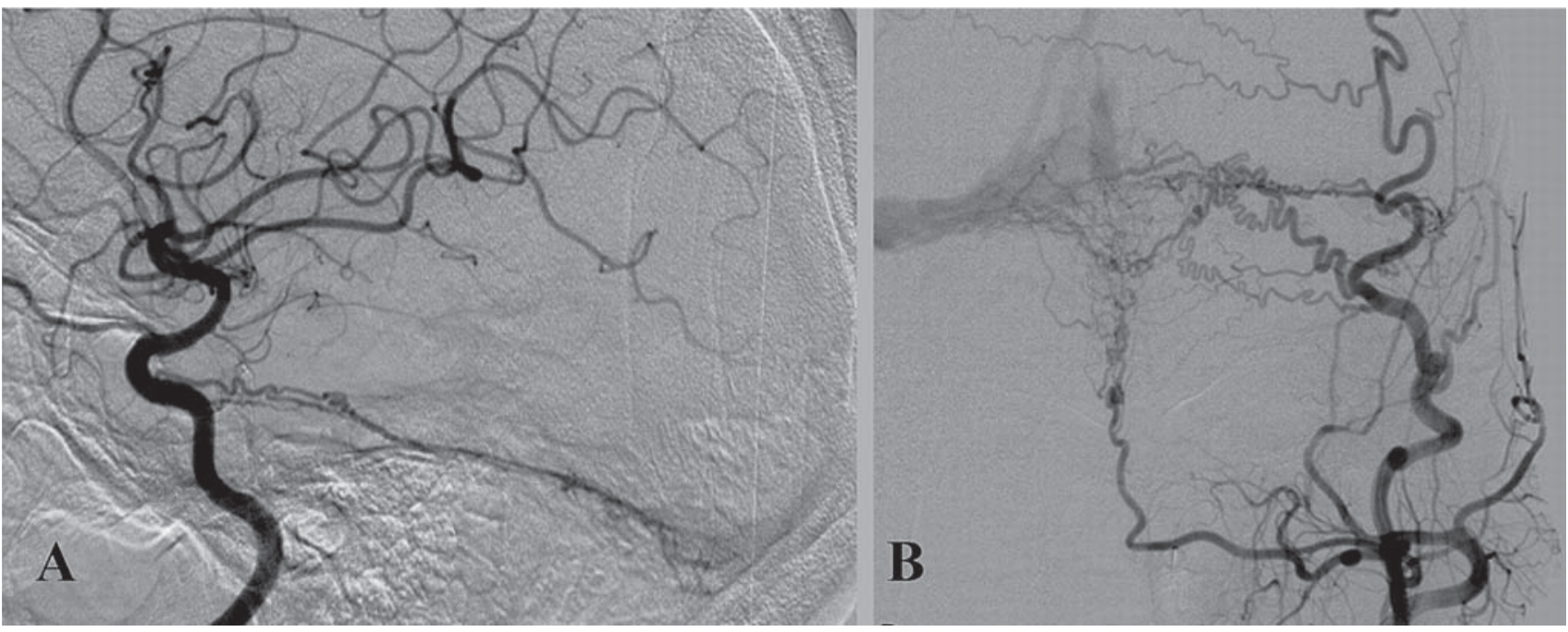

Figure 3: A) Another feeder is from reccurent meningeal brach of right ICA, B) selective left ECA angiogrpahy showing multiple small tortuos feeders from MMA

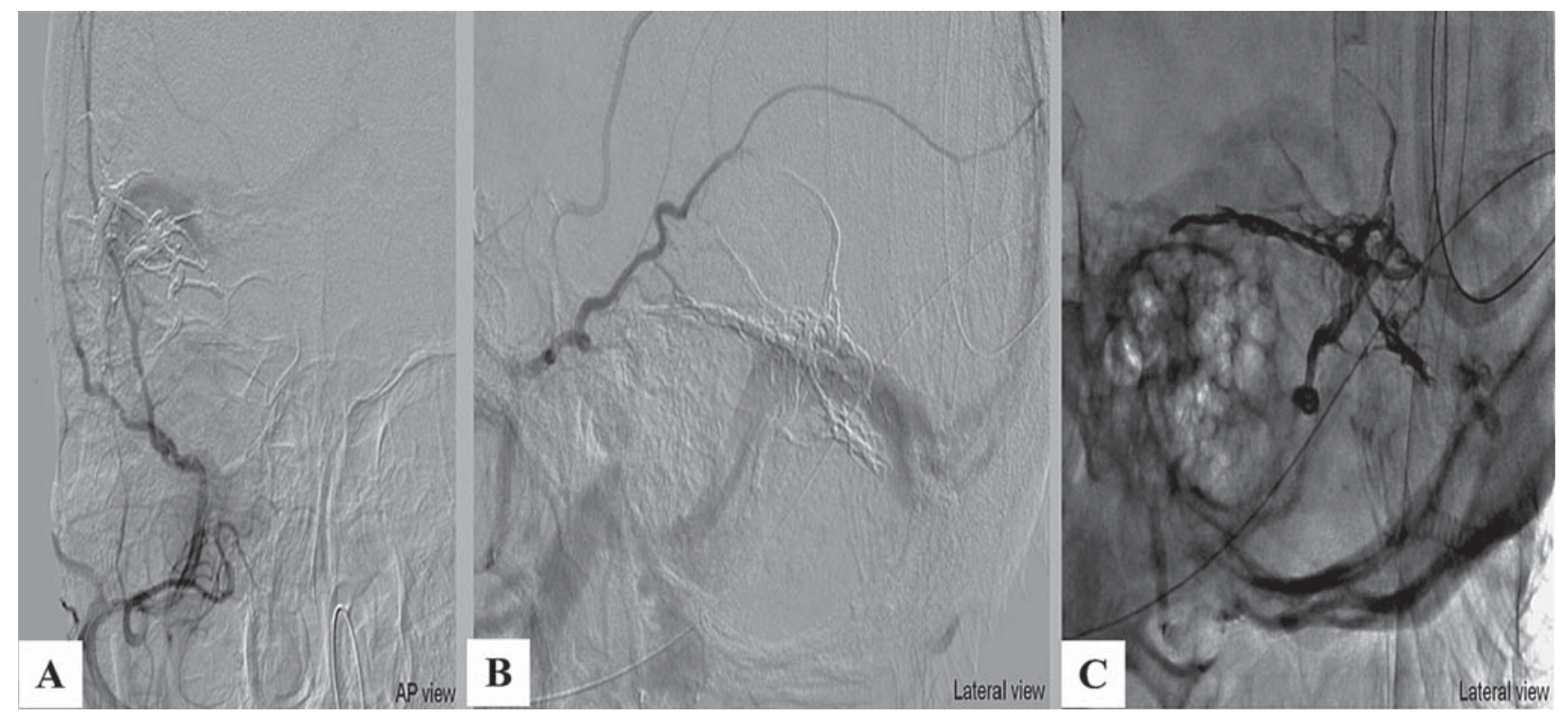

Figure 4: Selective right ECA angiography showing embolization with Onyx-18, DAVF is obliterated maximally from trans arterial approach via the branches of MMA, A) AP view, B) Lateral view, C) unsubtracted image lateral view shows cast of Onyx

local anesthesia, six-vessel angiography (bilateral ICA, ECA and VA) was performed via right femoral access. 5F standard cerebral angiographic catheter (VER $135^{\circ}$ Cordis) was selectively navigated into desired vessel. This study included at least three projections (anteriorposterior, lateral and oblique). For each projection, 2-4 $\mathrm{ml}$ of nonionic contrast bolus dose of Ioversol (320 $\mathrm{mg} / \mathrm{ml}$ ) was manually injected to obtain 3-4 frames per second to late venous phase. Magnification views were assimilated to elucidate significant findings. The fistulous site and its connections (e.g. arterial feeders, venous drainage pattern, collateral flows and dangerous vascular anastomosis) were well assessed. Based on location and characteristic hemodynamic changes in diagnostic angiography, embolization approach was considered after proper counselling.

\section{Embolization procedure for DAVF}

We performed embolization with ethylene vinyl alcohol EVOH (Onyx-18, ev3) particles under general anesthesia. 6-Fr guide catheter (Cordis Corp.) was placed at the origin of distal common carotid artery. Selective micro-catheterization of right ECA feeders was performed. Microcatheter was placed at the origin of feeding vessel to maintain good flow in the pedicle and avoid vasospasm induced by distal catheterization. Marathon 


\section{Karki et al}

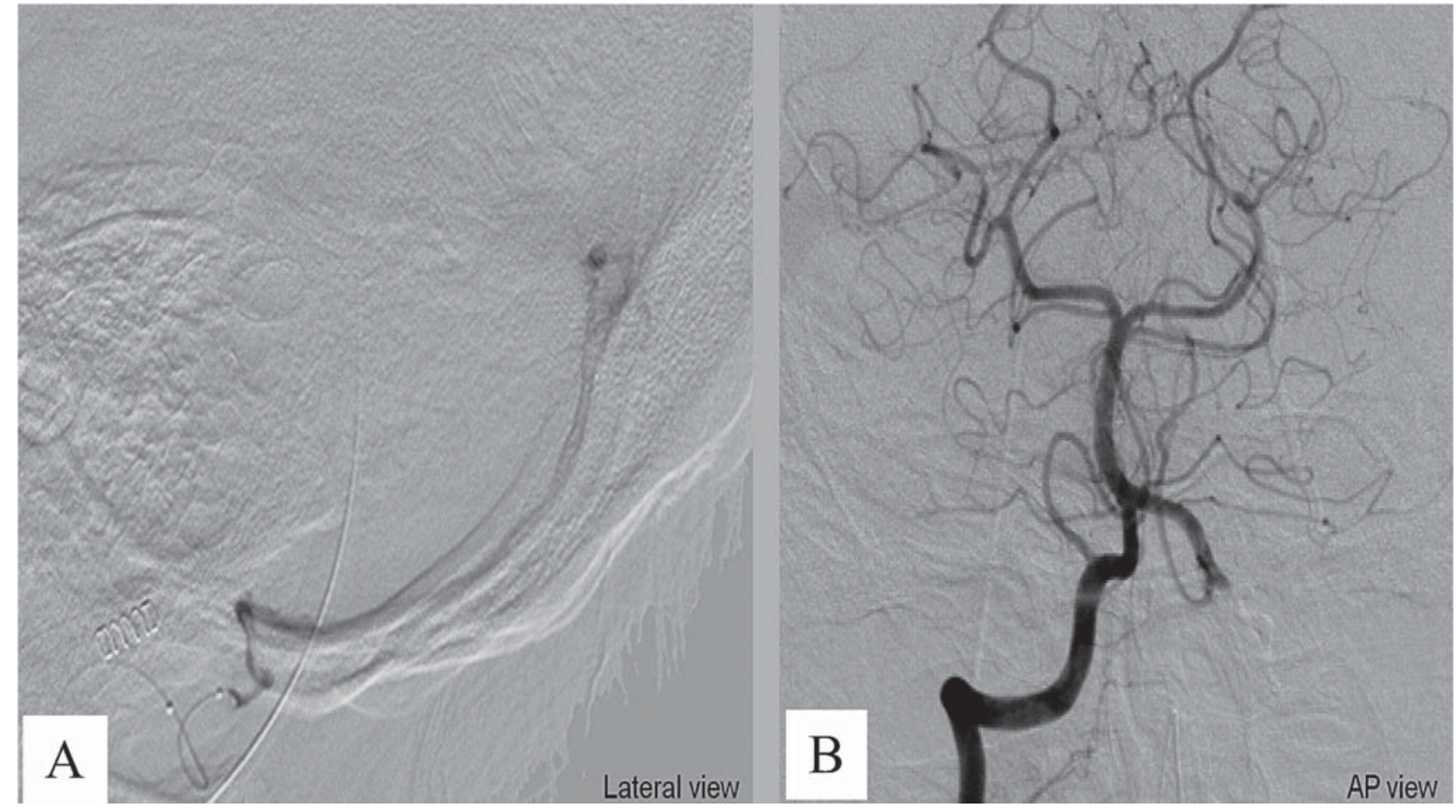

Figure 5: A) Superselective microcatheterization of posterior meningeal branch via left VA and injection of embolic material into the fistula, B) Selective right VA angiography after embolization, disappearance of fistula is noted

flow-directed catheter (ev3) was navigated over a micro guide wire (Transend series; Boston Scientific, eV3; or Mirage 008, ev3) to reach the distal aspect of the arterial pedicle supplying the fistula. Super-selective angiography was then performed to confirm optimal wedging of the microcatheter. Onyx-18 was slowly injected under real-time road-mapping. Whenever unwanted reflux or flow into an undesirable vessel was observed, injection was held for 20 seconds to 2 minutes to allowing Onyx polymerization, and reinjection was carried out slowly, in a controlled manner, engendering a new road map (refluxhold-reinjection technique). Maximum ECA feeders were embolized in same setting (Figure 4 A, B). Cast of onyx was well formed in non-subtracted image (Figure $4 \mathbf{C}$ ). With same procedure recurrent branch of meningeal supply from the left VA was also embolized (Figure 5 A) preserving posterior inferior cerebellar artery in both sides (Figure 5, B). Heparin was discontinued at the end of the procedure. Routine postoperative care was given in neurosurgery intensive care unit.

\section{Case 2}

A 23-year-old right handed healthy lady brought in casualty with complains of multiple episodes of generalized abnormal body movements for four days followed by loss of consciousness for an hour after each attack. Her conscious level gradually deteriorated and she remained in altered sensorium with loss of speech and paucity in motor activity in right side leading to difficulty in walking. Before throwing seizures, she had intermittent episode of mild to moderate headache in left side without complains of nausea, vomiting, and features of transient ischemic attacks.

On examination, she was disoriented with global aphasia. She had hemiparesis with power grade of 4 and plantar reflex was extensor on the right side. CT head showed diffuse tangle of vascular tumor in left parietal lobe with multiple tortuous abnormally dilated vessels (Figure 6A). Patient was started on anticonvulsants and admitted for supportive care in ICU. Patient underwent DSA. Angiography revealed grade V supratentorial AVM according to Spetzler Martin classification. There was huge diffuse nidus of size $6.4 \mathrm{~cm}$ occupying frontal parietal and occipital lobes. Major feeders were from middle cerebral artery (MCA), anterior cerebral artery (ACA) (Figure 6B) and posterior cerebral artery (PCA) on the left side. Lesion was draining majorly into superior sagittal sinus between anterior $1 / 3^{\text {rd }}$ and posterior $2 / 3^{\text {rd }}$ segment. High flow fistula was noticeable with retrograde filling of cortical veins (Figure 6C).

\section{Embolization procedure for giant AVM}

Embolization was performed under the general anesthesia. Systemic herparinization (4000 units IV bolus 

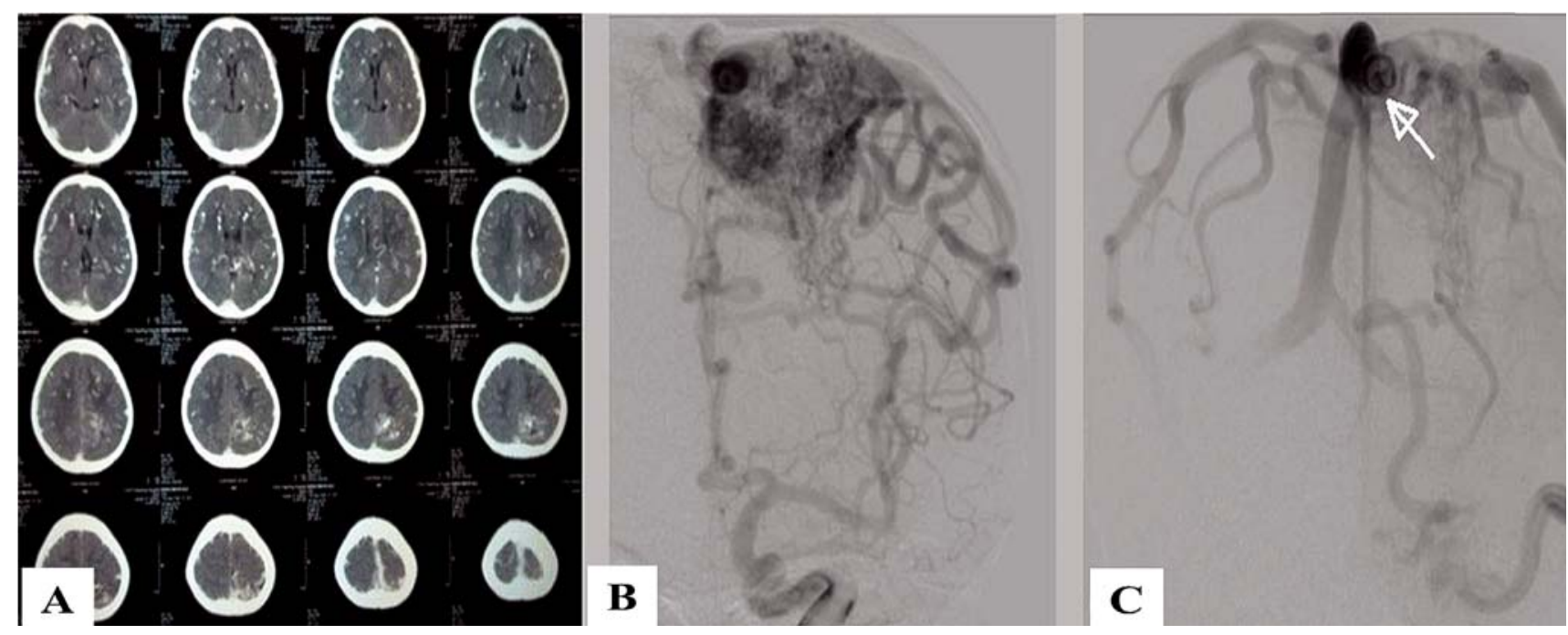

Figure 6: A) Non contrast CT scan shows multiple abnormal dilated vessels in bilateral hemisphere with foci of calcification in left posterior parietal lobe B) Selective left ICA arteriography showing AVM of > $6 \mathrm{~cm}$ size feeding by left MCA and ACA C) Early filling of draining veins into suprior sagital sinus with venous ectacia (white arrow) and presence of cortical venous reflux.

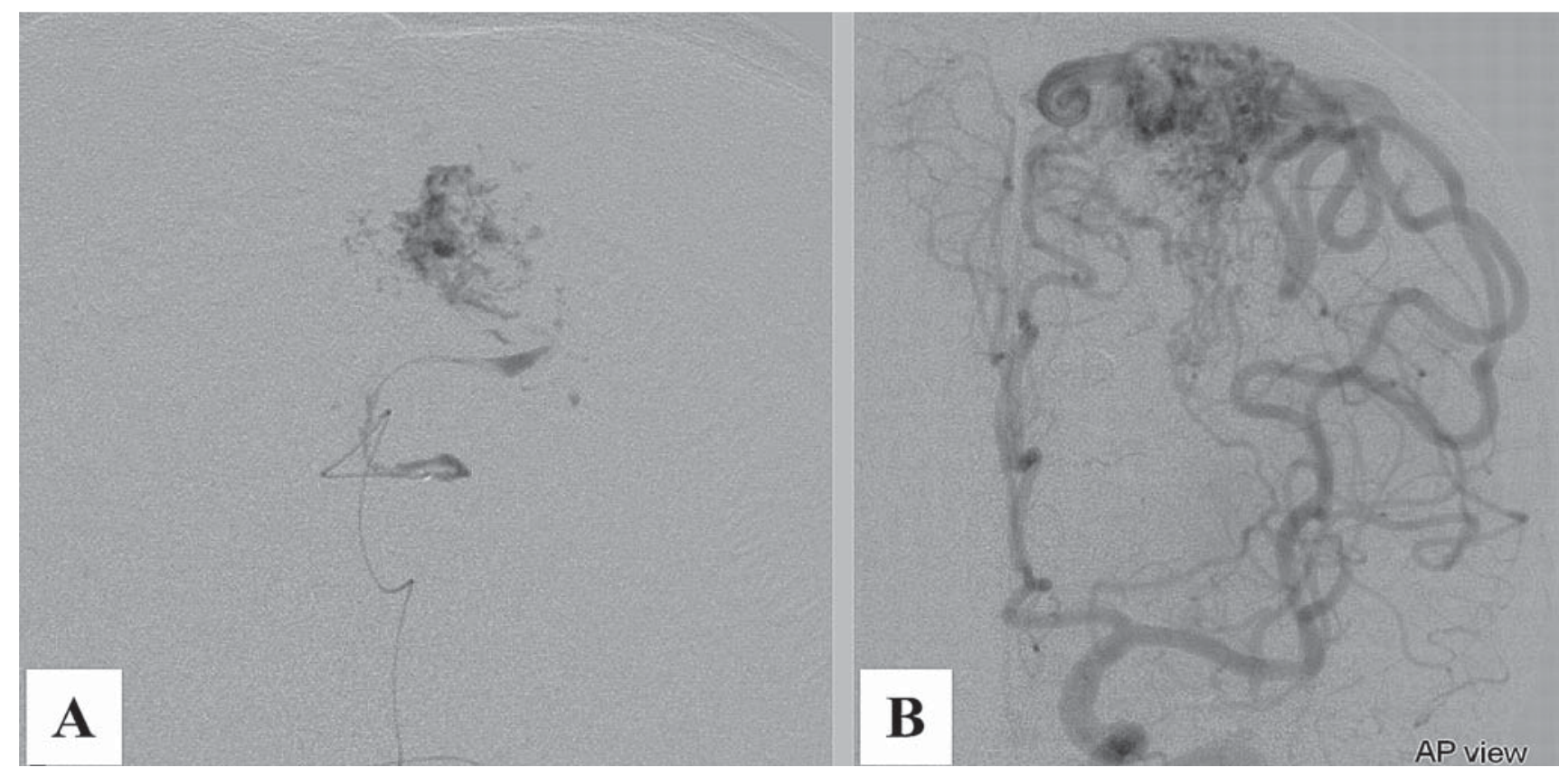

Figure 7: A) Placement of micro catheter near the nidus and injection of NBCA, B) Significant volume reduction of AVM with decrease in flow in fistulous connection between feeders and draining vein.

followed by half of the initial dose after each subsequent hours of treatment). All catheters used during the procedure were continuously flushed via the saline put into the pressure bag. 90 cm, 6 Fr guiding catheter (Cordis Corp.) was advanced at petrous segment of ICA for anterior approach and distal part of V4 segment of VA for posterior approach. Pre-embolization base line angiography was performed to assess for the interval change since the initial diagnostic angiogram and to confirm the selected approach to AVM. Flow directed 1.8-Fr Magic microcatheter (Target Therapeutics), Echelon series microcatheter (Micro Therapeutics) and hydrophilic micro guidewire (Transend series; Boston Scientific or Mirage 008, ev3) were used for the entire procedure. Once the tip of the microcatheter was positioned within the nidus or near the entrance of the fistula, super-selective angiography was performed to evaluate the flow characteristics, angioarchitecture and venous outflow of the nidus. Filling of contrast into the 


\section{Karki et al}

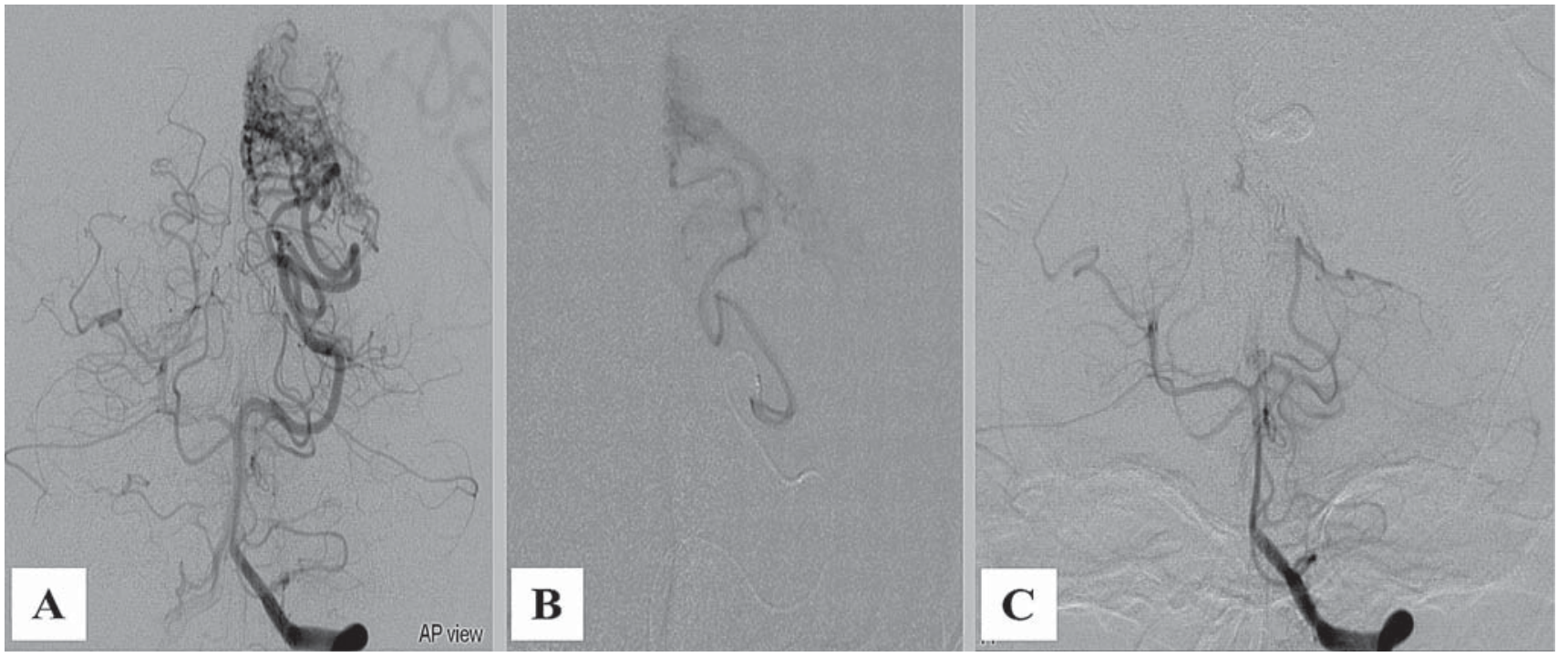

Figure 8: A)APview, selective left VA injection shows left PCAgiving offthe feeders, B) Superselective microcatheterization of left PCA and injection of NBCA, C) Complete obliteration of AVM demonstrated on VA injection (AP view)

nidus, contrast reflux, was determined. Optimal wedging of microcatheter was done after satisfactory super-selective angiography. The preparation of NBCA and lipiodol blend was decided according to the hemodynamic flow in arteriovenous shunt. The mixture of NBCA in ethiodol (25\%) was injected with flow controlled technique, filling the interstices of the nidus up to the draining vein (Figure 7A). Injection was continued until the proximal reflux of glue begins to extend long the microcatheter or until the nidus has filled and there is no progression of glue. Procedure was done 2 times from MCA and ACA (Figure $7 \mathbf{A}, \mathbf{B}$ ) and 1 time via the left PCA (Figure 8 A, $\mathbf{B}, \mathbf{C})$. Injection volumes were between $0.4-0.9 \mathrm{ml}$ and the injection time was between 1-2 minutes.

\section{Discussions}

World is undergoing a rapid revolution in the field of medical sciences. Amazing advances in technology has changed the vision in decision making in management of complex vascular diseases in neurosurgery. Technical advances in endovascular neurosurgery have afforded new alternatives in the treatment of complex vascular malformations like DAVF, CCF, giant AVM and VOGM. DSA plays a crucial role so called gold standard technique to ascertain the diagnosis of vascular malformation that emboldens for optimal management as well as for therapeutic management in same set up. Real time angiographic evaluation of all kinds of vascular malformations provides crucial information about the complex anatomy including location, size, feeding vessels, transition points and involving draining veins. Besides these findings, vascular architecture of the lesion is an important consideration when planning the treatment. DSA study also offers additional important information regarding associated intra-nidal aneurysms in AVM which may have impact on both natural history of the disease and difficulty of any anticipated surgical procedures. Determination of the hemodynamic properties of draining vein is another crucial factor for successful endovascular management. Flow velocity of arteriovenous shunt determines the safety of amount whether embolic material for injection.

Flow-directed and flow-assisted micro catheters have made navigation of intracranial vessels safer and have allowed more accurate delivery of embolic materials. Solid agents consist of polyvinyl alcohol particles, fibers, micro coils, and micro balloons. ${ }^{10,12}$ Liquid agents consist of cyanoacrylate monomers such as NBCA ( $N$-butyl cyanoacrylate), as well as polymer solutions such as ethylene vinyl alcohol (EVOH). ${ }^{10,12}$

\section{DAVF}

DAVF constitutes approximately $10-15 \%$ of all intracranial malformations, ${ }^{9}$ which mostly occurs over age 40 years. There is usually prominent draining vein that can be large and torturous leaving the fistulous site. Severity of DAVF is considered according to the venous drainage pattern. Venous varices may look like venous aneurysms. When fistula drains via superior ophthalmic vein, orbital and neuro-ophthalmologic symptoms are more prominent and with presence of leptomeningeal venous drainage, neurological findings or intracerebral hemorrhage is the primary clinical presentation. ${ }^{16}$

DAVF embolization can be done either via trans-venous or via trans-arterial route. ${ }^{10}$ Trans-arterial embolization for 


\section{Cerebrovascular Lesion}

is an ideal technique for permanent fistulous occlusion and to preserve patency of draining venous sinuses primarily for ncsDAVF obliteration and trans-venous approach is ideal for csDAVF. ${ }^{13,2,5}$ Trans-arterial technique includes super-selective feeding vessel catheterization and delivery of embolic material into dural branches of the fistula. Non cavernous type DAVF (ncsDAVF) typically drains directly into leptomeningeal veins or into major large dural venous sinuses, like transverse sinus and sigmoid sinus. Optimal patency of this venous drainage is mandatory during embolization. If not it may change venous outflow into other sinuses, or even into cortical veins resulting into intracranial hemorrhage. ${ }^{10,8}$ Complete fistulous obliteration was obtained via the trans-arterial approach without reflux into any involved sinuses. ${ }^{10,14}$ Inadequate dispersion of embolic material into all fistulous connections may occur and pedicles of arterial feeders could prematurely be occluded, which could ultimately result in fistula remnants and recurrence. ${ }^{17,7}$ Onyx has better penetrating quality than in other embolic materials. Complete occlusion rate after trans-arterial embolization of ncsDAVF with polyvinyl alcohol (PVA) particles, NBCA or a combination of them was only $27.3-30 \% .^{7}$ In our case, embolization was done via three feeders. MMA was the most commonly approached vessel. Cortical venous reflux with dilated venous pouch was retrograded into superior sagittal sinus. Although the ECA feeders were longer and tortuous, super selective catheterization was successfully done into right posterior branch of MMA and near complete occlusion was achieved. Due to presence of rich connection network between fistulas and arterial feeders, Onyx penetrated well into all fistulous connecting networks, even though the smaller and single artery branch from high to low pressure, till all connections were completely occluded. Due to its non-adhesive character, repeated injection for longer duration is allowed, providing sufficient control over its tapping to prevent outflow deep into the venous circulation or into other normal vasculature.

\section{AVMs}

Brain AVMs are intraparenchymal conglomerations of dilated arteries and veins lacking normal vascular structures resulting in direct connections between the cerebral artery and veins without intervening capillary bed. They present as either focal or diffuse and complex vascular lesions that can result in significant morbidity and mortality. On angiography, AVMs demonstrate arteriovenous shunting resulting in early opacification of draining veins and shortens arteriovenous transit time. Embolization is an important component in the treatment of patients with AVM. It can be an essential adjunct to surgery or radiosurgery affecting eloquent areas like basal ganglia thalamus Rolandic cortex. Less often, embolization is done as primary therapy for smaller but surgically difficult lesions. ${ }^{3}$ Implementation endovascular therapy for AVM has been categorized into preoperative, intraoperative, preradiosurgery, curative and palliative embolization. ${ }^{15}$ Aim of embolization of giant AVM is either curative or palliative. Prerequisite for curative treatment of AVM by endovascular method is presence of nidus that can hold solid cast material in it. Curative embolization is defined as complete anatomical obliteration of nidus and early venous shunting. Permanent non-biodegradable embolic material is thus required for better penetration and organization of solid cast within the nidus. Curative embolization is possible if the malformation is smaller in size with maximum 1- 2 vascular pedicles. However with advance technical development of endovascular therapy in embolization of vascular malformation, stage embolization has been in practice for curative purpose with better results. ${ }^{4}$ Palliative embolization usually does not remove the risk of rebleed. The rationale for partial embolization includes reduction in size of lesion which is usually indicated in inoperable AVM. Palliative embolization is also done to reverse a progressive neurological deficit. In such case venous pressure reduction or control of venous hypertension is the actual goal. After partial embolization, anatomical cure can be accomplished by a combination of two or more modalities. Multimodality treatment can minimize the risk and improve clinical outcome. For multimodality treatment of AVM, role of pre-operative embolization has become well established. ${ }^{1}$ Pre-surgical embolization facilitates good surgical removal by decreasing operative time, blood loss and thus reduces morbidity and mortality rates. Hence embolization has become imperative in treatment plan in developed countries for gaining maximum therapeutic benefit. Adjuvant embolization is performed for stage reduction of blood flow in the AVM by stepwise occlusion of nidus over a period of time. Obliteration of deep feeders like lenticulostriate arteries which could require extensive resection plane beyond the margin of nidus and obliteration of skull base dural feeders which are not readily accessible during surgical resection can be included in adjuvant embolization. Hence preoperative embolization in large AVM shows advantage for progressive thrombosis stabilizing local and regional hemodynamic changes. ${ }^{11}$

\section{Conclusions}

DSA remains the study of choice for precise interpretation of dynamic angioarchitecture of various neurovascular diseases and subsequently to elaborate 


\section{Karki et al}

on the potential treatment protocols. Embolization with liquid embolic material can be an integral part of the multidisciplinary treatment protocol for patients with complex vascular malformations. Endovascular therapy in Nepal has many constraints. This outset will have positive impact in the country that has to deal with moderate resources to provide wide therapeutic benefit.

\section{References}

1. Baskaya MK(1), Jea A, Heros RC, Javahary R, Sultan A. Cerebral arteriovenous malformations. Clin Neurosurg 53:114-144, 2006

2. Blount J P, Oakes W J, Tubbs R S, Humphreys R P. History of surgery for cerebrovascular disease in children. Part II. Vein of Galen malformations. Neurosurg Focus 20:E10, 2006

3. Bruno CA Jr, Meyers PM. Endovascular management of arteriovenous malformations of the brain. Interv Neurol 1:109-123, 2013

4. Conger A, Kulwin C, Lawton MT, Cohen-Gadol AA. Endovascular and microsurgical treatment of cerebral arteriovenous malformations: Current recommendations. Surg Neurol Int 6:39, 2015

5. Ellis JA, Lavine SD. Role of embolization for cerebral arteriovenous malformations. Methodist Debakey Cardiovasc J 10:234-239, 2014

6. Heros RC. Multimodality treatment of cerebral arteriovenous malformations: modern treatment of cerebral arteriovenous malformations World Neurosurg 82:46-48, 2014

7. Kirsch M, Liebig T, Kuhne D, Henkes $H$. Endovascular management of dural arteriovenous fistulas of the transverse and sigmoid sinus in 150 patients. Neuroradiology 51:477-483, 2009

8. Kiyosue H, Hori Y, Okahara M, Tanoue S, Sagara Y, Matsumoto S, et al. Treatment of intracranial dural arteriovenous fistulas: current strategies based on location and hemodynamics, and alternative techniques of transcatheter embolization. Radiographics 24:1637-1653, 2004

9. Klisch J, Huppertz HJ, Spetzger U, Hetzel A, Seeger W, Schumacher M. Transvenous treatment of carotid cavernous and dural arteriovenous fistulae: results for 31 patients and review of the literature. Neurosurgery 53:836-856, 2003

10. Long XA, Karuna T, Zhang X, Luo B, Duan CZ: Onyx 18 embolization of dural arteriovenous fistula via arterial and venous pathways: preliminary experience and evaluation of the short-term outcomes. Br J Radiol 85:395-403, 2012

11. Martin NA, Khanna R, Doberstein C, Bentson J. Therapeutic embolization of arteriovenous malformations: the case for and against. Clin Neurosurg 46:295-318, 2000

12. OgilvyCS, Stieg PE,AwadI, BrownRDJr, Kondziolka D, Rosenwasser R, Young WL, Hademenos G. Recommendations for the management of intracranial arteriovenous malformations: a statement for healthcare professionals from a special writing group of the Stroke Council, American Stroke Association. Stroke 32:1458-1471, 2001

13. Parsa AT, Solomon RA. Vascular malformations affecting the nervous system. In: Rengachary s, ellenbogen

14. Piske RL, Campos CM, Chaves JB, Abicalaf R, Dabus G, Batista LL, et al. Dural sinus compartment in dural arteriovenous shunts: a new angioarchitectural feature allowing superselective transvenous dural sinus occlusion treatment. AJNR Am J Neuroradiol 26:1715-1722, 2005

15. Potts MB, Zumofen DW, Raz E, Nelson PK, Riina HA. Curing arteriovenous malformations using embolization. Neurosurg Focus 37(3):E19, 2014

16. Quinones D, Duckwiler G, Gobin PY, Goldberg RA, Vinuela F. Embolization of dural cavernous fistulas via superior ophthalmic vein approach. AJNR Am J Neuroradiol 18:921-928, 1997

17. Wakhloo AK, Perlow A, Linfante I, Sandhu JS, Cameron J, Troffkin N, et al. Transvenous N-butylcyanoacrylate infusion for complex dural carotid cavernous fistulas technical considerations and clinical outcome. AJNR Am J Neuroradiol 26:1888-1897, 2005 